\title{
Energy Efficient Nodes Clustering and Routing Using Multi-Objective Spider Monkey Optimization Algorithm in Wireless Sensor Network
}

Duraimurugan S ( $\nabla$ duraimurugans2021@gmail.com )

St Joseph Engineering College

Avudaiammal $\mathbf{R}$

St Joseph Engineering College

\section{Research Article}

Keywords: Wireless sensor network (WSN), load balancing, clustering, routing and spider monkey optimization (SMO)

Posted Date: July 20th, 2021

DOl: https://doi.org/10.21203/rs.3.rs-618230/v1

License: (c) (1) This work is licensed under a Creative Commons Attribution 4.0 International License.

Read Full License 


\title{
Energy Efficient Nodes Clustering and Routing Using Multi- Objective Spider Monkey Optimization Algorithm in Wireless
}

\section{Sensor Network}

\author{
${ }^{1}$ Duraimurugan $\mathrm{S},{ }^{2} \mathrm{R}$. Avudaiammal \\ ${ }^{1}$ Associate Professor \\ Department of Information Technology \\ St. Joseph's College of Engineering \\ Chennai, Tamil Nadu, India \\ duraimurugans2020@gmail.com \\ ${ }^{2}$ Professor \\ Joseph's College of Engineering, \\ Chennai, India
}

\begin{abstract}
In wireless sensor network (WSN), the gateways which are placed far away from the base station (BS) forward the collected data to the BS through the gateways which are nearer to the BS. This leads to more energy consumption because the gateways nearer to the BS manages heavy traffic load. So, to overcome this issue, loads around the gateways are to be balanced by presenting energy efficient clustering approach. Besides, to enhance the lifetime of the network, optimal routing path is to be established between the source node and BS. For energy efficient load balancing and routing, multi objective based spider monkey optimization (SMO) algorithm is presented in this paper. Using this algorithm, optimal clustering and routing are performed
\end{abstract}


depend on the objective functions routing fitness and clustering fitness. Simulation results show that the performance of the proposed SMO based clustering and routing scheme attains better results than that of the existing algorithms in terms of energy consumption, delivery ratio, throughput and network lifetime.

Keywords: Wireless sensor network (WSN), load balancing, clustering, routing and spider monkey optimization (SMO).

\section{Introduction}

WSNs must refer to a group or network in an accepting state [1] [2]. The various devices on the network are mean by sensor nodes [3] [4]. The sensor node collects data from the environment and transmits it to the base station (BS) via a central station or a direct wireless connection. WSN is used to monitor environmental, national boundaries. [5] A strategy in which each sensor node communicates directly with the BS. To change this, neural ganglia are organized into different groups. Each node has a communication channel between the sensor and the BS device. The button on the sensor that opens the button is called the cluster head $(\mathrm{CH}) . \mathrm{CH}$ contact details of the places of hearing in the Rights Group and their BS identification. WSNs that follow this strategy are collectively known as WSNs.

Another strategy to reduce communication efforts is to use Gateway [6] [7]. The gate is a device with a comparatively large insulating battery that acts like a $\mathrm{CH}$ [8] [9]. Use of the gateway prevents rapid loss of electricity through $\mathrm{CH}$. Remote BS Ports sends data to the nearest BS Gateway. As a result, holes near the BS carry more traffic loads and consume more of their energy quickly, which increases the problem of container release containers around the BS 
[10].If there is a power gap around the BS station, the network is disconnected and BS loses the connection to the remote gate. This increases the need for network load balancing management [11] to avoid the power gap problem and extend network life. To address these problems, it is necessary to present the circuit diagrams in this article to clusters and redirect custom-enabled nodes.

Contributions of this paper are described as follows:

$>\quad$ This document presents the ideal design of a spider monkey optimization (SMO) which has many purposes for collecting electricity and energy.

$>\quad$ Lastly The last thing you can do is reduce the total power consumption and you will pay for the best route from the gate to the BS. The power function of the group is selected in such a way that the gate has a different number of BS sensory areas. The latter solution reduces the power consumption of the gate and sensors.

The proposed method used is used in the MATLAB platform area.

This proposed method performance tested for power consumption, network life, power measurement and performance.

The whole document is organized as follows. Section 2 reports some previous articles that focused on collecting and conducting trail research at WSN. Section 3 proposes the collection and distribution of energy saving areas using a multi-objective SMO algorithm at WSN.

\section{Related works}

In this section, the survival of some earlier texts focuses on merging and integrating routing and routing in WSN. A. Sundar Rajina and M. Chinadurai [12] suggested that network operations be 
managed using a load saver system with a opportunistic energy-efficient routing with load balancing (OE2-LB) that eliminates data collection delays and avoids data collection. Move loops efficiently with smart wearable points. Thereafter, system performance is verified for network life, delays, error metrics, energy efficiency, and network mobility.

Reeta Bhardwaj and Dinesh kumar [13] presented a Multi-objective fragmentary molecule lion calculation (MOFPL). The proposed MOFPL figure finds a suitable team leader in various WSN locations. At that point, the appropriate approach is established according to the proposed multipurpose capability. The proposed MOFPL value consists of 5, 8, 10 nodes active during WSN 2000 pressures of 50, 75, and 100 nodes, each. Similarly, the proposed MOFPL calculation found high system stability of 0.05877 and 0.06022 WSN at 50 and 100 nodes, each on the network.

Faouzi Hidowssi et al [14] proposed a new conference for PEAL (Power Efficient and Adaptive Latency) wireless networks. Our retrospective results show that PEAL can extend system life by $47 \%$ compared to the LEACH (Low Energy Adaptive Clustering Hierarchy) model meeting and show a delay in better mobility compared to energy protection gains. .

Yasha istwal and Shashi kant Verma [15] have been able to have paper centers that will reduce energy consumption, improve reliability, service life and system performance. From the replay, it can be seen that the DCHRP4 review period is $56.11 \%$ on the election protocol (ETSSEP) and twice and more than thrice over edge sensitive stable election protocol (TSEP) and stable election protocol (SEP). each. The life expectancy of DCHRP4 was also revised 8.29\% and 49.22\% in ETSSEP and TSEP individually, while almost three times in the SEP trial. Performance improved by $7.75 \%$ over ETSSEP, doubling TSEP and SEP respectively. 
Nehad A Marsey, Ehab H.Abdel Hay and Sherif S.Kishk[16] suggested that the CH selection process be organized as a single development goal aimed at achieving a positive $\mathrm{CH}$ formation, the collections return., adjusting the use of firmness, stiffness and deceleration using gravitational search algorithm (GSA). This problem was solved using the GSA measure and the molecular model and the outcome of the LEACH meeting. In addition, a new cost-saving function has been proposed for group groups. The purpose of motivating the group by building the health sector is to build a healthy lifestyle and increase program stability; Some hobbies are designed to monitor the performance of various escape routes.

Seyed Naser Hashemi and Ramin Yarinezhad [17] had proposed a scale algorithm to solve the problem with an average rating of 1.1. This algorithm operates a controlled time for fixed parameters. We use the base of the visible lattice in the system, which creates a pragmatic algorithm for powerful WSNs. In addition, we propose a channel algorithm based on this structure. The router algorithm reduces and balances system power consumption by finding the appropriate routes between each group head and sink. The entertainment results show that the proposed algorithm is a pragmatic for powerful WSNs despite having a high display and other similar algorithms.

Y.U, Hao et al [18] proposed to find a well thought-out solution with a wolf pack algorithm (WPA) that would improve aircraft performance and performance, while the strange communication algorithm based on the advanced improved wolf pack algorithm (LWPA) was updated. Also, to address the LWPA algorithm communication method problem, the edge degree concept often builds the DEEC algorithm. The improved DEEC algorithm (IDEEC) is used to gradually integrate a common node in complex systems, and a data transfer mode is created after the integration mode is configured. Finally, with the experimental findings, compared to other 
network router algorithms, the CLWPA algorithm effectively delays the stability and service life of the network, and the energy efficiency is measured.

Damodar Reddy Edla ,Amruta Lipare and Venkatanareshbabu Kuppili [19] had proposed using the Gray Wolf Optimization (GWO) method to consolidate power and lead WSN. Additionally, we suggest two new and advanced skills for sharing and managing challenges. The steering function aims to maintain a normal decrease in cracks and the number of bounces in the range. Combined health work carries an overall burden, as indicated by the separation of entry routes to BS. The proposed GWO-based approach is based on a high degree of integration and compliance with statistics, specifications, current asset calculations, multicellular bias, and complex integration.

\section{Energy Efficient Nodes Clustering and Routing in WSN}

\subsection{Overview}

For enhancing the energy efficiency and network lifetime, optimal load managing and routing are to be presented in WSN. To attain these aims, multi-objective based spider monkey optimization (SMO) algorithm is presented in this paper. For energy efficient routing, an optimal path is selected between gateways and base station (BS) using the SMO algorithm. It will helps to reduce the overall energy consumption in the network. For load balancing, the SMO algorithm uses a clustering format function and selects the solution such that gateways near the BS are assigned a relatively smaller number of sensor nodes and gateways outside More BS sensor nodes are assigned from the BS.

\subsection{System model}


Figure 1 shows the system model of WSN. This model includes four clusters and one BS. Each cluster includes gateway $(\mathrm{G})$ and cluster members. As shown in the figure, these cluster members are connected to the corresponding $G$ in each cluster. The function of load distempering gateways in WSN is illustrated schematically in Figure 2. In Figure 2, gateways G1, G2, G3 and G4 correspond to 4 different clusters. As can be seen in Figure 2, seven sensor nodes are connected to G1, while two sensor nodes are present in G2 has three, G3 has three and G4 has four sensor nodes. Therefore, the G1 gateway loads more than G2, G3 and G4. Consequently, the energy consumed by G1 is greater than G2, G3 and G4. Higher energy consumption shortens the life of the G1. This results in poor network performance and reduced overall network life. If G1 fails, G2 share the load of G1 as shown on the right side of the figure 2. It can also cause premature death of G2, G3 and G4. A scientific approach to cluster load, load, and routing is necessary to prevent such problems with the WSN.
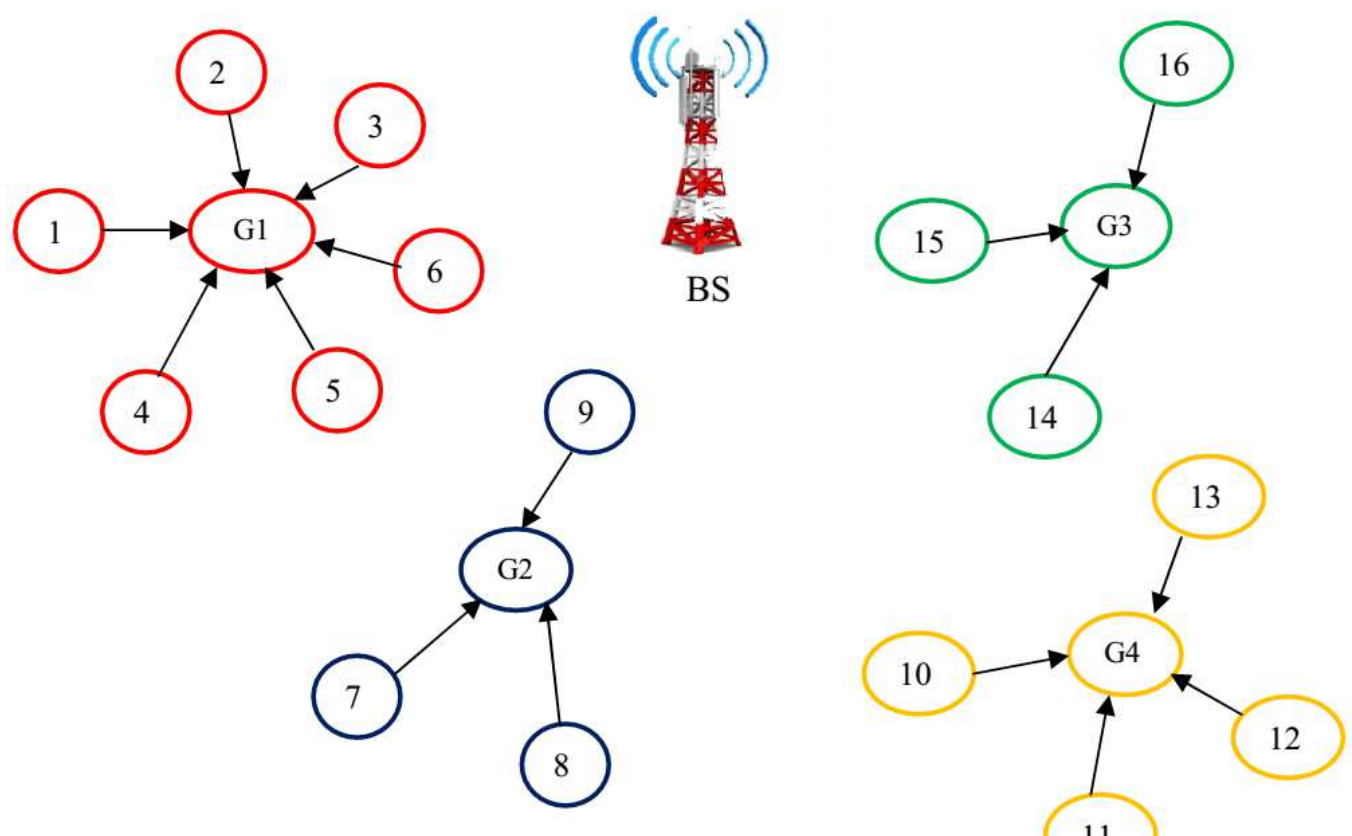

11

Figure 1: System model of WSN 

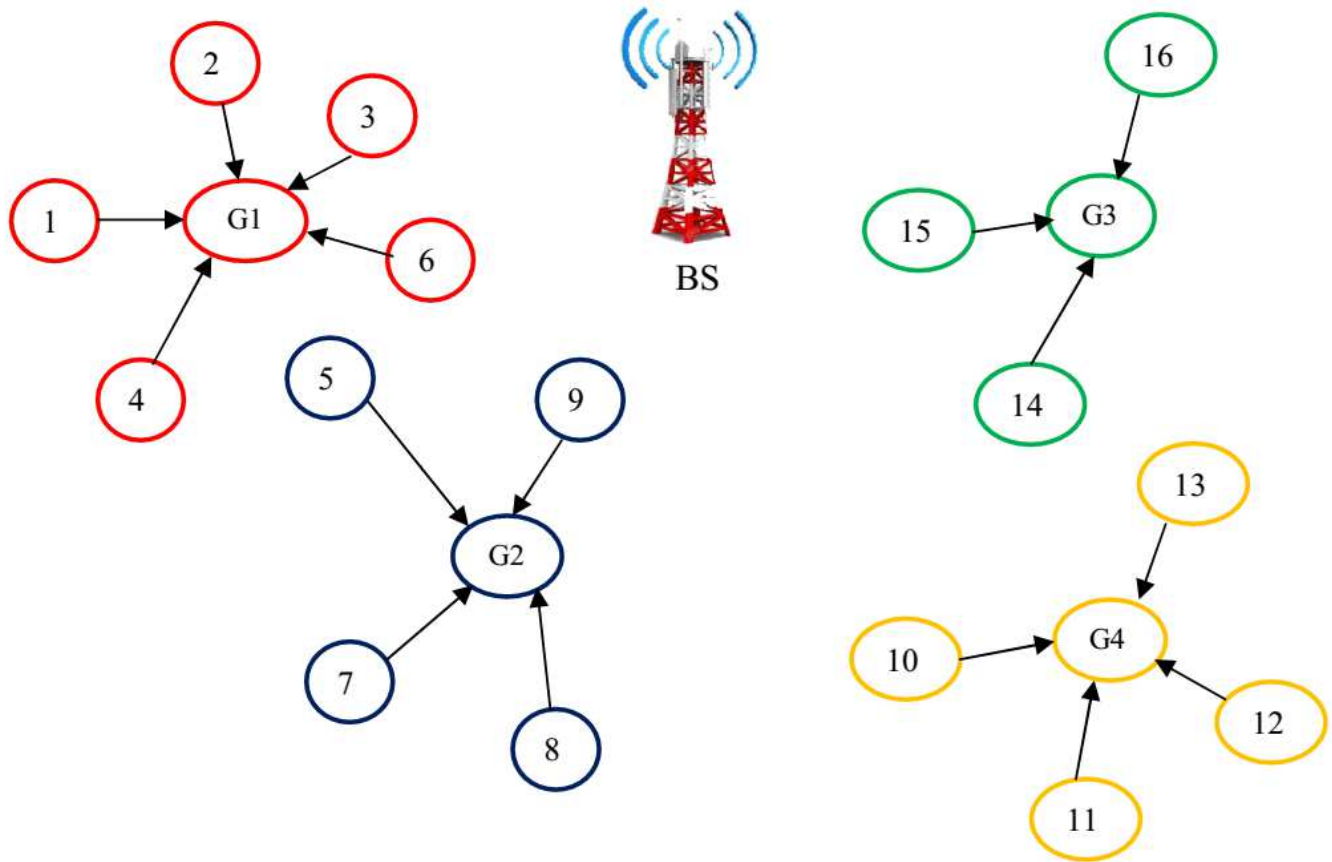

Figure 2: Load balancing in WSN

\subsection{Energy Efficient Routing using SMO algorithm}

This document introduces the spider monkey optimization (SMO) algorithm for optimal integration and load balancing. The SMO algorithm incorporates the practice of searching for food for spider monkeys. These behaviors can be divided into four categories according to the social fission-fusion social structure (FFSS) structure of spider monkeys. First, the group checks the food classification and begins eating the food. In the second step, the positions of food collection and distribution are reviewed. In the latter case, the local pioneer restores his good standing in the group. Everyone in the group began to review the diet due to the lack of renewal of the excellent position by the group pioneer. In the fourth step, the international pioneer 
reopens his good position. This group was divided into smaller groups because of the position (no increase in overseas pioneers in the period described earlier). The local pioneer limit (LPL) has been linked to the reduction of the problem-solving event by turning the circle into a harvest theme. On the other hand, the meeting is divided into smaller groups if the international pioneer is able to review the global pioneer limit (GPL). Negative reviews are received from the LPL and GPL of local and international pioneers to make their own decisions.

Initialization: The position $(\mathrm{P})$ of each solution or spider monkey is represented in the form of a map from one $\mathrm{G}$ to another or BS. This solution provides access to the operating system from each gateway through the following network gateways. Each gateway starts with a random number $\left(\mathrm{U}_{\mathrm{i}, \mathrm{d}}\right)=\operatorname{rand}(0,1)$ where $1 \leq \mathrm{i} \leq \mathrm{Ms}, 1 \leq \mathrm{d} \leq \mathrm{T}$. $\mathrm{M}_{\mathrm{s}}$ is the number of major solutions. Gateway numbers are the solution to component $d$. Set the $\mathrm{G}_{\mathrm{k}}$ gateway to the BS route as the next gateway to $G_{d}$, indicating that $G_{d}$ is sending data to $G_{k}$. The mapping of the routing path is formulated in equation (1)

$$
G_{k}=I\left(\operatorname{SetNextg}\left(G_{k}\right), m\right)
$$

Where $I\left(\operatorname{SetNextg}\left(G_{k}\right), m\right)$ is the face work by decreasing face of $m^{\text {th }}$ gateway from SetNextg and $\left.m=\operatorname{Ceil}\left(U_{i, d} \times \mid \operatorname{SetNextg}\left(G_{k}\right)\right)\right)$

Fitness calculation: After the initialization of solutions, fitness value is calculated for each solution. The fitness of routing is specified to generate efficient routes from each gateway to the BS. Routing path is selected by minimum distance and minimum number of hops.

$$
\text { Fit }_{\text {Routing }}=\operatorname{Max}\left(\frac{C_{1}}{D_{\text {Total }} * \mathcal{W}_{1}+G_{\text {Hop }} * \mathcal{W}_{2}}\right)
$$

Where, $\left(\mathrm{w}_{1}, \mathrm{w}_{2}\right) \in[0,1]$ such that, $\mathrm{w}_{1}+\mathrm{w}_{2}=1$ and $\mathrm{C}_{1}$ is a proportionality constant. $\mathrm{D}_{\text {Total }}$ represents the total distance traversed by gateways is defined as follows 


$$
D_{\text {Total }}=\sum_{i=1}^{n} D\left(G_{i}, N \operatorname{extg}\left(G_{i}\right)\right)
$$

Besides, $\mathrm{G}_{\mathrm{Hop}}$ taking the total number of the gateway hops of the networks

$$
G_{H o p}=\sum_{i=1}^{n} \operatorname{Nextg} \operatorname{Count}\left(G_{i}\right)
$$

According to equation (2), the solution with maximum fitness function is considered as the optimal solution or optimal routing path.

Update the solution: This renewal phase includes the following categories, such as the local pioneer class, the international pioneer class, the worldwide pioneer training class, the local pioneer dependence section, the worldwide pioneer decision-making section, and the pioneer decision-making phase. of this place. The components of this algorithm are described below.

Local pioneer phase: At this point, each spider or monkey determines its new position based on the experience of local pioneers and local team members.

$$
P_{\text {new } i, d}=\left\{\begin{array}{lr}
P_{i, d}+r_{1} \times\left(L P_{k, d}-P_{i, d}\right)+r_{2} \times\left(P_{j, d}-P_{i, d}\right) & \text { if } r_{1} \geq P R \\
P_{i, d} & \text { otherwise }
\end{array}\right.
$$

There, $r_{1}$ and $r_{2}$ represent the random numbers $[0,1]$ and $[-1,1], L P_{k}, d$ represents the position of the local pioneer in $\mathrm{k}^{\text {th }}$ magnitude. $\mathrm{d}^{\text {th }}$ represents the spider monkey randomly selected in group $\mathrm{P}_{\mathrm{j}}, \mathrm{d}$ on the $\mathrm{j}^{\text {th }}$ scale $\mathrm{d}^{\text {th }}$. PR represents a measure of certain factors that indicate the amount of perturbation.

Global pioneer phase: The local pioneer class is followed by this taking, known as the world pioneer class. on the point, all the spider monkeys update their positions based on the experiences of local team members and international leaders.

$$
P_{\text {new }}=P_{i, d}+r_{1} \times\left(G P_{d}-P_{i, d}\right)+r_{2} \times\left(P_{j, d}-P_{i, d}\right)
$$


There, $G P_{d}$ represents the position of international pioneers in the $d^{\text {th }}$ dimension. Spider monkeys are revived according to the following number of possibilities. This can include job stress. The solution or the spider monkey is more likely to reuse its position. This number of opportunities $\left(P r_{i}\right)$ is calculated as follows:

$$
\operatorname{Pr}_{i}=\frac{\text { Fit }_{i}}{\max -\text { Fit }} \times 0.9+0.1
$$

Learning phase of global pioneer: At this stage, the position of overseas pioneers is being reviewed, and a powerful spider monkey has been selected as an international pioneer. If the levels of pioneers overseas are not reviewed, the international border value of a good local solution will increase by 1 .

Learning phase of local pioneer: Given the role of the local leader, the local limit on the number of pilot land solutions will increase by 1 .

Decision phase of local pioneer: If the number of local leaders exceeds the number of local leaders, the status of all team members is determined at random or based on information received from local and foreign leaders.

$$
P_{n e w_{i, d}}=P_{i, d}+r_{1} \times\left(G P_{d}-P_{i, d}\right)+r_{1} \times\left(P_{i, d}-L P_{j, d}\right)
$$

From equation (8), it is cleared that the position of the spider monkey is updated towards the position of global pioneer.

Decision phase of global pioneer: At this phase, the population is divided into smaller groups where the border is larger than the international pioneer limit. A local pioneer limit is set for selecting a local pioneer from each group. If the position of the pioneers abroad is not reviewed, the international pioneer will join all the groups and form a group. 
Termination: Above described phases are continued until find the optimal solution or the best channel frequency response $\left(\operatorname{Best}\left(\hat{\boldsymbol{H}}_{n, k}^{L S}\right) \& \operatorname{Best}\left(\hat{\boldsymbol{H}}_{n, k}^{M M S E}\right)\right)$ from the initialized channel estimators. If the optimal solution is attained simultaneously for LS and MMSE channel estimation techniques, then the algorithm is terminated.

Termination: The process is repeated until the optimal solution or routing path obtained. Then result is attained, the algorithm will be terminated.

\subsection{Energy Efficient Nodes clustering using SMO algorithm}

The SMO-based merging algorithm uses the solution found in the proposed route algorithm. The base station makes a set of gate sensors to measure the load on the gates using the route solution found in the proposed algorithm. We use a different integration algorithm because the dynamic channel is not suitable for group applications.

Initialization: Clustering algorithm, says the size of the solution corresponds to the number of sensors $(\mathrm{N})$. In the first method, all sensor space is assigned a random number $\left.\mathrm{V}_{\mathrm{i}, \mathrm{d}}\right)=\operatorname{rand}(0,1)$ where $1 \leq \mathrm{i} \leq \mathrm{M}_{\mathrm{c}}, 1 \leq \mathrm{d} \leq \mathrm{N}$. $\mathrm{M}_{\mathrm{c}}$ is the number of initial clustering solutions. Compound $\mathrm{D}$ contains the index of the sensor node (s) and the corresponding inputs to the gateway are in the same cluster as the GK cluster head, indicating that $S_{d}$ sends data to the $G_{k}$. The mapping of the sensor node and gateway is expressed in equation (9).

$$
G_{k}=I\left(\operatorname{Coms}\left(S_{d}\right), m\right)
$$

Where $I\left(\operatorname{Coms}\left(S_{d}\right), m\right)$ is an indexing function which returns index of nth gateway from the set of $\operatorname{Coms}\left(S_{d}\right)$ and $m=\operatorname{Ceil}\left(V_{i, d} \times \mid \operatorname{Coms}\left(S_{d}\right)\right)$. Since the volume of the solution is equal to the number of sensor nodes, the introduction and disassembly of the nodes results in a difference in the solution size necessitating the need for reassembly. 
Fitness calculation: After initialization, a new fitness grouping function is evaluated for each solution. The closest gateways to the BS are connected to the fewest sensor nodes, while the remote gateways are connected to the most sensor nodes. Gateway outside BS sends data from gateway outside BS. Thus the gateway minimizes its own load as it is connected to a low sensor node.

$$
\text { Fit }_{\text {Clustering }}=\operatorname{Min}\left(\frac{\sum_{i=1}^{n} \text { Fit }_{\text {Clustering }}\left(G_{i}\right)}{n}\right)
$$

Where, Fit Clustering $\left(G_{i}\right)$ represents the clustering fitness function for the gateway and is calculated as follows

$$
\text { Fit }_{\text {Clustering }}\left(G_{i}\right)=C_{2} \times\left|L\left(G_{i}\right)-D\left(B S, G_{i}\right) * \mu\right|
$$

Where, $\mathrm{C}_{2}$ denotes the proportionality constant, $\mu$ represents the mean load over all gateways according to the distance between gateways and BS. This mean load $(\mu)$ is calculated as follows:

$$
\mu=\frac{\sum_{i=1}^{n} L\left(G_{i}\right)}{n}
$$

Update the solution: The solution is updated as the updation is done in routing phase and the updation is continued until finding the optimal solution using equation (5), (6) and (8).

Termination: The solution is updated until an optimal solution or clustering is found. The algorithm will terminate as soon as the optimal solution is found. Fig. 3 illustrates the SMO algorithm for clustering and routing schemes. 


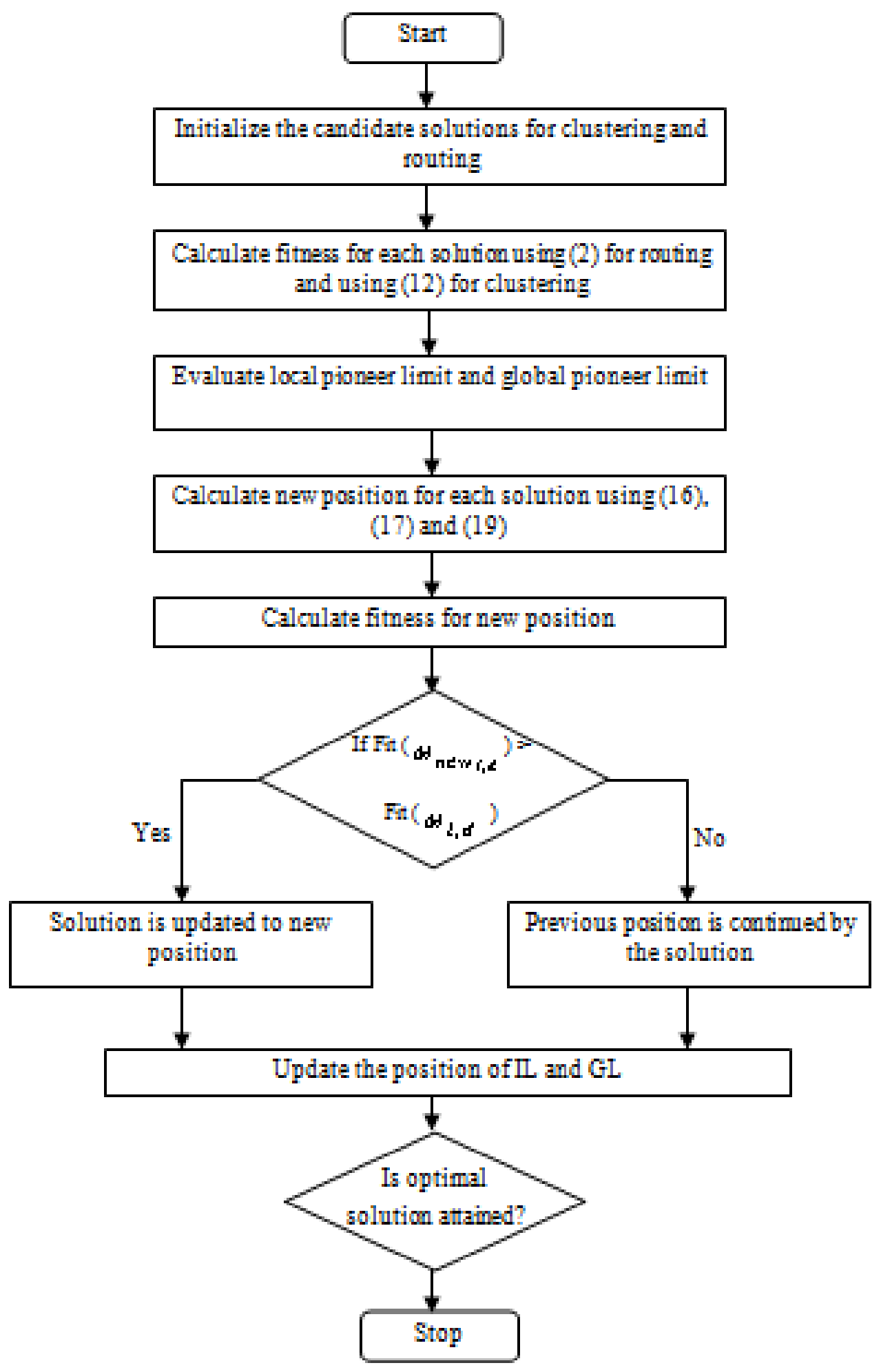

Figure 3: Flowchart of multi-objective SMO algorithm 


\section{Results and Discussions}

The proposed program is being implemented on the Matlab platform. In this experiment 50 meters are operated in an area of 50 meters. Each sensor node can transmit the power up to the distance of 250 meters. The AODV routing protocol is used for this purpose. Each sensor node is equipped with a light-emitting tenta that produces the same radio frequency energy in all directions.

Table 1: Simulation setting

\begin{tabular}{|c|c|}
\hline Parameter & Value \\
\hline Area size & $1000 \mathrm{~m} \times 1000 \mathrm{~m}$ \\
\hline Routing protocol & AODV \\
\hline MAC & $802 \_11$ \\
\hline Antenna & Omni Antenna \\
\hline Radio propagation model & Two Ray Ground \\
\hline Packet size & $512 \mathrm{bytes}$ \\
\hline Initial transmitting power & $0.660 \mathrm{~W}$ \\
\hline Initial receiving power & $0.395 \mathrm{~W}$ \\
\hline Initial energy & $10.3 \mathrm{~J}$ \\
\hline
\end{tabular}

\subsection{Performance analysis}

The proposed performance in clustering and routing scheme using SMO is evaluated based on the number of sensor nodes in delay, distribution ratio, power consumption, throughput and network lifetime. In addition, the functionality of the SMO algorithm is compared with existing PSO and GWO algorithms. Fig. 4 shows comparison of delay of different schemes for different nodes. As shown in the figure, the delay increases as the number of sensor nodes increases. 
However, the proposed SMO-based clustering and root plan are 21\% and 37\% lower than GWO and PSO, respectively.. As the SMO algorithm has good convergence speed and less computational complexity than the GWO and PSO, optimal clustering and routing have been done efficiently. Due to the efficient load balancing with the efficient clustering algorithm, transmission delay of the data packet is reduced.

The comparison between delivery ratio of the proposed SMO and that of the existing PSO and GWO is shown in figure 5. The figure represents the increase in the sensor node number. However, the optimal route selection using the proposed SMO resulted in an $8 \%$ and $29 \%$ increase in the delivery rate of the proposed scheme compared to the existing GWO and PSO. Figure 6 shows a comparison of the energy consumption of the clustering and routing scheme with different algorithms. The energy consumption of the proposed SMO has been reduced by $6 \%$ and $33 \%$ respectively as compared to GWO and PSO due to the proposed clustering scheme and the choice of better route using load balancing at the entrance.

As shown in the figure, throughput is decreased when the number of sensor nodes increases. However, compared to existing GWO and PSO working clustering and routing scheme, throughput of the given SMO based clustering and routing scheme is increased to $61 \%$ and $72 \%$ respectively. As the SMO algorithm has good convergence speed, routing path between source and BS is selected optimally than the existing GWO and PSO. So, it leads to increase the throughput of the network.

However, network lifetime of the proposed SMO based clustering and routing scheme is increased to $21 \%$ and $37 \%$ than that of existing GWO and PSO based clustering and routing schemes respectively. Because of the load balancing among the gateways using the proposed SMO based clustering scheme, network lifetime of the network is increased. 


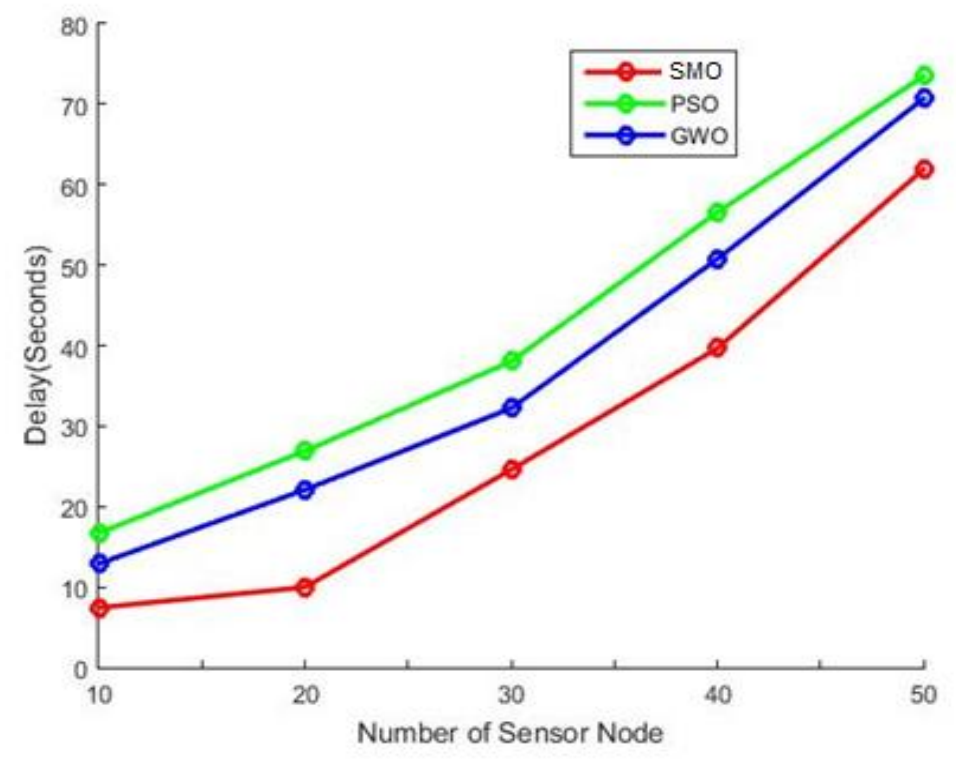

Figure 4: comparative analysis of different approaches in terms of Delay

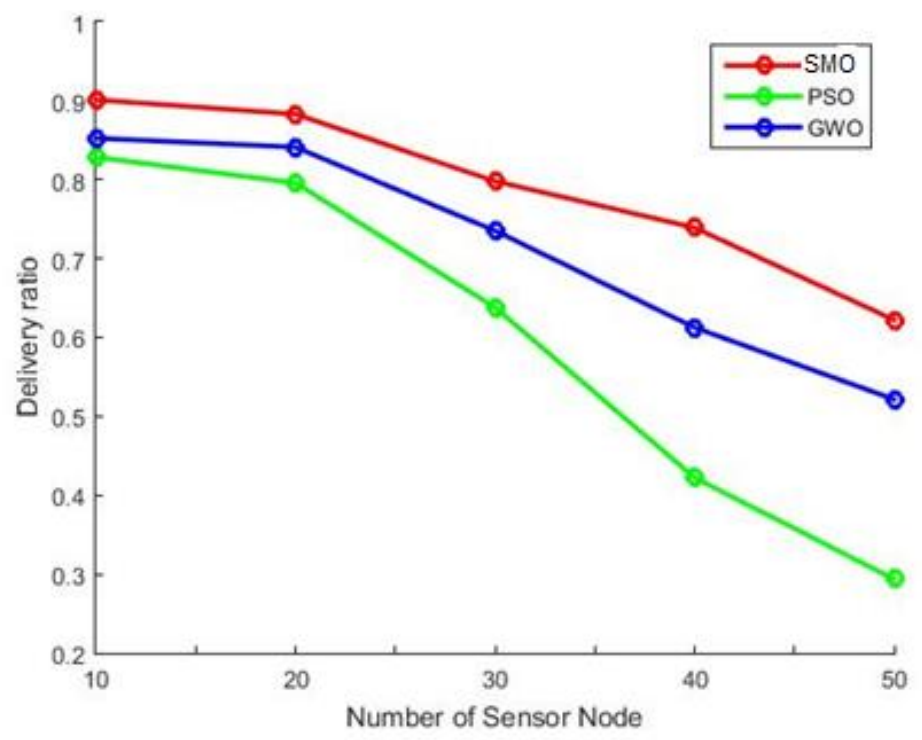

Figure 5: comparative analysis of different approaches in terms of Delivery ratio 


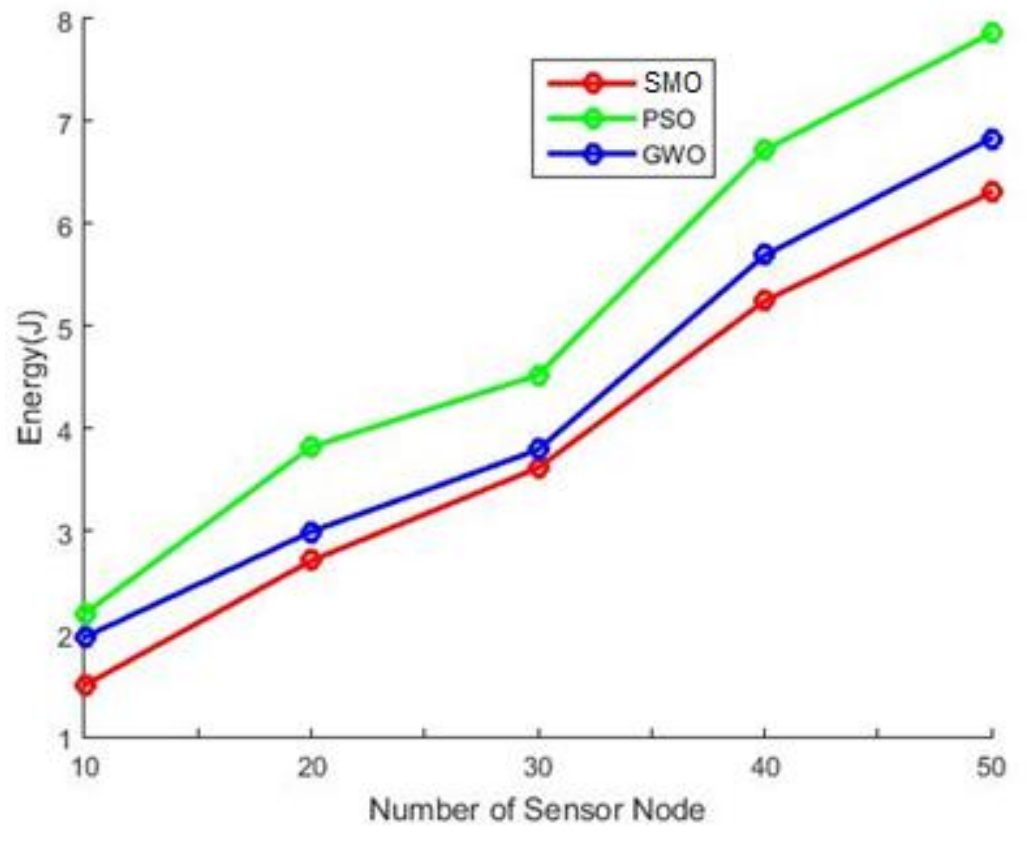

Figure 6 comparative analyses of different approaches in terms of Energy consumption

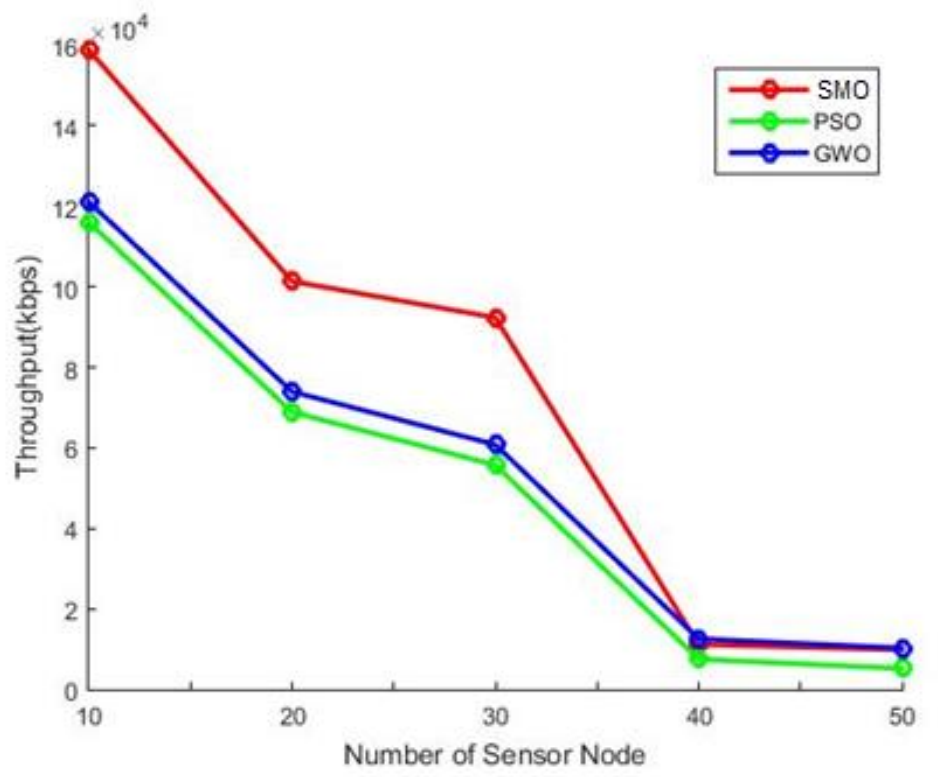

Figure 7: comparative analysis of different approaches in terms of Throughput 


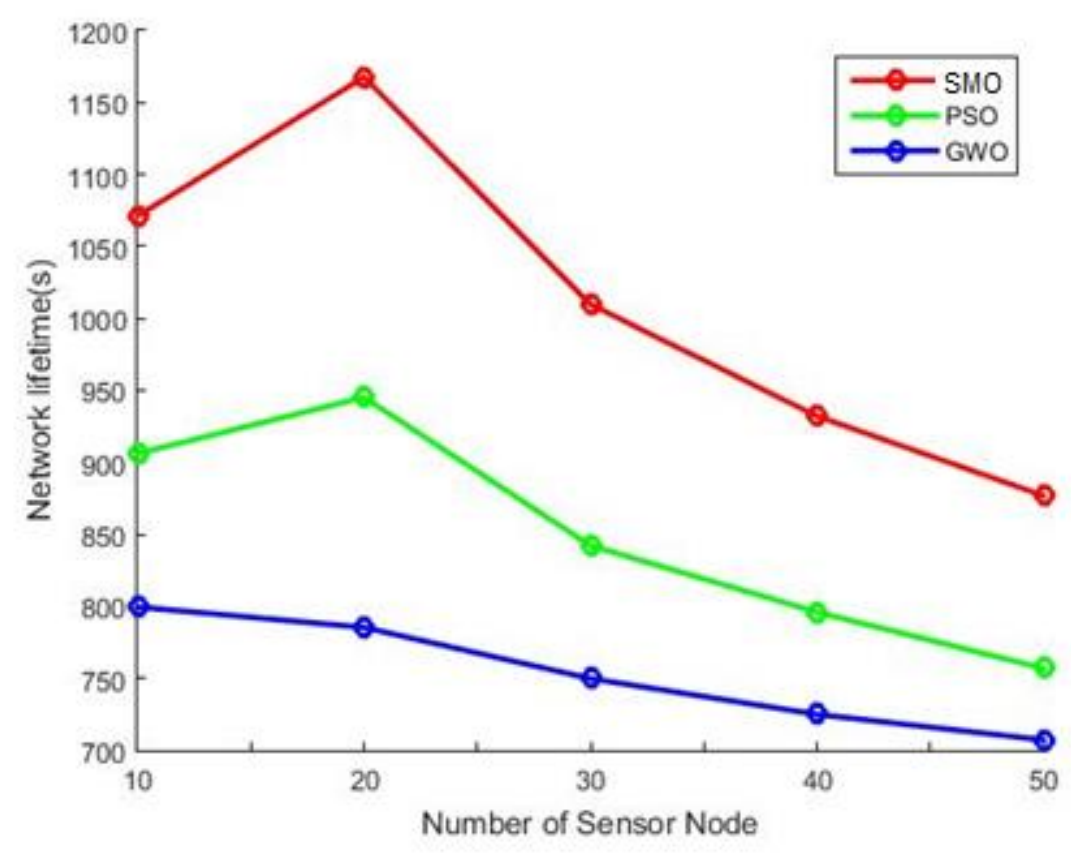

Figure 8: comparative analysis of different approaches in terms of Network lifetime

\section{Conclusion}

By considering the major issues such as heavy traffic load around the gateways and network lifetime in WSN, multi-objective spider monkey optimization (SMO) algorithm based clustering and routing scheme has been presented in this paper. Using the SMO based clustering the sensor nodes are assigned to the gateway near the BS and a large number of sensor nodes are assigned to the gateway away from the BS way. In addition, to increase the energy efficiency and network life of the network, the optimal route between the source and the BS is established using the SMO algorithm.. The proposed SMO based clustering and routing scheme outperformed the existing PSO and GWO based clustering and routing schemes in terms of network lifetime and energy consumption. 


\section{References}

[1].Raghavendra, Cauligi S., Krishna M. Sivalingam, and Taieb Znati, eds. Wireless sensor networks. Springer, 2006.

[2].Sankarasubramaniam, Y., I. F. Akyildiz, W. Su, and E. Cayirci. "Wireless sensor networks: A survey." Computer Networks 38, no. 4 (2002): 393-422.

[3].Rao, Bhawna, and Ms Anjali Saxena. "Role Of Sensor Nodes In Wireless Sensor Network." IJIRMPS-International Journal of Innovative Research in Engineering \& Multidisciplinary Physical Sciences 6, no. 2 (2018).

[4].Merrett, Geoff V., Bashir M. Al-Hashimi, Neil M. White, and Nick R. Harris. "Resource aware sensor nodes in wireless sensor networks." In Journal of Physics: Conference Series, vol. 15, no. 1, p. 137. IOP Publishing, 2005.

[5].Tang, Jian, Bin Hao, and Arunabha Sen. "Relay node placement in large scale wireless sensor networks." Computer communications 29, no. 4 (2006): 490-501.

[6].Kuila, Pratyay, Suneet K. Gupta, and Prasanta K. Jana. "A novel evolutionary approach for load balanced clustering problem for wireless sensor networks." Swarm and Evolutionary Computation 12 (2013): 48-56.

[7]. Kuila, Pratyay, and Prasanta K. Jana. "Energy efficient clustering and routing algorithms for wireless sensor networks: Particle swarm optimization approach." Engineering Applications of Artificial Intelligence 33 (2014): 127-140.

[8].Edla, Damodar Reddy, Amruta Lipare, Ramalingaswamy Cheruku, and Venkatanareshbabu Kuppili. "An efficient load balancing of gateways using improved shuffled frog leaping algorithm and novel fitness function for WSNs." IEEE Sensors Journal 17, no. 20 (2017): 6724-6733. 
[9].Edla, Damodar Reddy, Amruta Lipare, and Ramalingaswamy Cheruku. "Shuffled complex evolution approach for load balancing of gateways in wireless sensor networks." Wireless Personal Communications 98, no. 4 (2018): 3455-3476.

[10]. Mohemed, Reem E., Ahmed I. Saleh, Maher Abdelrazzak, and Ahmed S. Samra. "Energy-efficient routing protocols for solving energy hole problem in wireless sensor networks." Computer Networks 114 (2017): 51-66.

[11]. Chen, Guihai, Chengfa Li, Mao Ye, and Jie Wu. "An unequal cluster-based routing protocol in wireless sensor networks." Wireless Networks 15, no. 2 (2009): 193-207.

[12]. Raj, A. Sundar, and M. Chinnadurai. "Energy efficient routing algorithm in wireless body area networks for smart wearable patches." Computer Communications 153 (2020): 85-94.

[13]. Bhardwaj, Reeta, and Dinesh Kumar. "MOFPL: Multi-objective fractional particle lion algorithm for the energy aware routing in the WSN." Pervasive and Mobile Computing 58 (2019): 101029.

[14]. Hidoussi, Faouzi, Homero Toral-Cruz, Djallel Eddine Boubiche, Rafael Martínez-Peláez, Pablo Velarde-Alvarado, Romeli Barbosa, and Freddy Chan. "PEAL: Power efficient and adaptive latency hierarchical routing protocol for cluster-based WSN." Wireless Personal Communications 96, no. 4 (2017): 4929-4945.

[15]. Istwal, Yasha, and Shashi Kant Verma. "Dual cluster head routing protocol with super node in WSN." Wireless Personal Communications 104, no. 2 (2019): 561-575.

[16]. Morsy, Nehad A., Ehab H. AbdelHay, and Sherif S. Kishk. "Proposed Energy Efficient Algorithm for Clustering and Routing in WSN." Wireless Personal Communications 103, no. 3 (2018): 2575-2598. 
[17]. Yarinezhad, Ramin, and Seyed Naser Hashemi. "Solving the load balanced clustering and routing problems in WSNs with an fpt-approximation algorithm and a grid structure." Pervasive and Mobile Computing 58 (2019): 101033.

[18]. Xiu-wu, Y. U., Y. U. Hao, Liu Yong, and Xiao Ren-rong. "A clustering routing algorithm based on wolf pack algorithm for heterogeneous wireless sensor networks." Computer Networks 167 (2020): 106994.

[19]. Lipare, Amruta, Damodar Reddy Edla, and Venkatanareshbabu Kuppili. "Energy efficient load balancing approach for avoiding energy hole problem in WSN using Grey Wolf Optimizer with novel fitness function." Applied Soft Computing 84 (2019): 105706. 